\title{
Food Industry
}

National Cancer Institute

\section{Source}

National Cancer Institute. Food Industry. NCI Thesaurus. Code C16590.

The businesses that raise, process, and distribute foodstuffs. 